\title{
Structure and thermal expansion of the low-temperature phase of $\mathrm{SF}_{6}$
}

\author{
A. P. Isakina and A. I. Prokhvatilov \\ B. Verkin Institute for Low Temperature Physics and Engineering, 47 Lenin Ave,, 61164 Kharkov, Ukraine \\ E-mail: isakina@ilt.kharkov.ua \\ J. Rodriguez-Carvajal \\ Laboratory Leon Brillouin, CEA Saclay, 91191 Gif-sur-Yvette Cedex, France
}

Received November 12, 1999, revised December 6, 1999

\begin{abstract}
Powder x-ray and neutron diffraction studies of the crystalline structure, lattice parameters and thermal expansion coefficients of sulphur hexafluoride $\mathrm{SF}_{6}$ are performed in the temperature range $1.64-110 \mathrm{~K}$. It is shown that the low-temperature phase (at $T<94.3 \mathrm{~K}$ ) is monoclinic, space group $\mathrm{C} 2 / \mathrm{m}\left(C_{2 h}^{3}\right)$ with $Z=6$ in which $1 / 3$ of molecules $\mathrm{SF}_{6}$ occupy the positions of higher symmetry $(2 / \mathrm{m})$ and $2 / 3$ of molecules - lower one $(m)$. As follows from the analysis of obtained structural results, an availability of two types of molecular local symmetry positions is responsible for the anisotropic character of molecular rotation, peculiarities on the temperature dependences of structural and thermodynamic properties of $\mathrm{SF}_{6}$ crystals at low-temperature phase, especially near $T_{c}$ of orientational phase transition. It is carried out a detail comparison the present results with the known literature data.
\end{abstract}

PACS: 61.10.-i, 61.12.-q, 61.50.Ks, 61.50.Em, 65.70.+y, 65.50.+m

\section{Introduction}

Sulphur hexafluoride $\mathrm{SF}_{6}$ crystallizes into a cubic bcc lattice of space symmetry $\operatorname{Im} 3 m\left(O_{h}^{9}\right)$ at $222.4 \mathrm{~K}$. As the temperature is decreased, there occurs a polymorphic transformation at $94.5 \mathrm{~K}$ which results in further orientational ordering of the molecules and decrease of the crystal symmetry. The orientational structure of the high-temperature phase ( $\beta$-phase) has been studied by $x$-ray $[1,2]$, neutron diffraction [3] and electron diffraction [4-6] techniques. It is found that in the vicinity of the phase transition the molecular S-F bonds are mainly oriented along the direction $\{100\}$ of the bcc lattice. But the orientational motion of the molecules in this phase is characterized, particularly at high temperatures, by high librations (the deviation of the S-F bonds from the direction along the lattice axes may be as much as $20^{\circ}$ ) and fast re-orientations, resulting in a high degree of dynamic disorder. As follows from $[7,8]$, the disorder results from the fundamental difference in the interaction with the first- and second-nearest neighbors, producing a frustration of the orientational structure of the high-temperature phase of $\mathrm{SF}_{6}$ [9].
The data on crystal structure of the low-temperature phase of $\mathrm{SF}_{6}$ are quite contradictory. The results given in $[3,4,6,10,11]$ even suggest that the orientational ordering in $\mathrm{SF}_{6}$ occurs by stages and is followed by the formation of an intermediate, partially ordered phase. According to the electron diffraction data $[4,5]$ obtained on the films, this phase is a hexagonal one of rhombohedral symmetry $P 3 m 1\left(D_{3 d}^{3}\right)$ at temperatures from 50 to $94 \mathrm{~K}$. At $T<50 \mathrm{~K}$ a slight phase distortion to a base-centered monoclinic lattice was also observed. The intermediate rhombohedral phase was also obtained by molecular-dynamics (MD) simulations [10,11]; in this structure only $2 / 3$ of the molecules are orientationally ordered. At the same time, neutron powder experiments $[7,8,12]$ have demonstrated the existence of only one low-temperature phase, with a triclinic lattice $P \overline{1}(Z=3)$. The neutron diffraction studies by Cockcroft and Fitch (1988) [13] favor the view that there is no second phase transition at $T \approx 50 \mathrm{~K}$. According to their data, the low-temperature phase of $\mathrm{SF}_{6}$ has a monoclinic lattice of the space group $C 2 / m\left(C_{2 h}^{3}\right)$ with six molecules per unit cell. 
In that context, we reasoned that a reexamination of low-temperature experiments using combined high-resolution neutron and x-ray diffraction studies of $\mathrm{SF}_{6}$ should be performed to elucidate conclusively the structure of the low-temperature phase and to obtain the data still unavailable, on vibrational anharmonicity and molecular rotational motion dynamics. The solution of the latter problems has been made possible by our $\mathrm{x}$-ray dilatometric measurements of the linear and volume expansion coefficients within the 4.2-94 K temperature range and the analysis of thermodynamic characteristics.

\section{Experimental procedure}

The neutron powder studies were carried out at the Laboratory Leon Brillouin (LLB), Saclay (France), on a G42 high-resolution spectrometer using radiation at wavelengths of $1.979 \AA$ and $2.596 \AA$ (from the (115) and (004) planes, respectively, of the Ge monochromator) at several temperatures in the range $1.64-110 \mathrm{~K}$. The angular resolution of the spectrometer was discussed in detail in Ref. 14. The samples were prepared by condensing $99.99 \%$ pure $\mathrm{SF}_{6}$ gas in a closed vessel at liquid-nitrogen temperature. In this process a snow like mass was formed which was then used to fill the cylindrical specimen chamber. Thereafter the chamber was quickly placed into a helium cryostat vessel, which was mounted on the spectrometer and preliminary cooled down to $1.5 \mathrm{~K}$. The minimum temperature of the neutron powder measurements was $1.64 \mathrm{~K}$. The sample temperature at each point was stabilized with an accuracy of $\pm 0.01 \mathrm{~K}$. The experiments made it possible to collect and analyze the neutron diffraction data in the reflection angle range from $2 \Theta$ to $158^{\circ}$ $\left(Q_{\max }=6.3 \AA^{-1}\right)$ with a $0.02^{\circ}$ step size scan.

The $x$-ray measurements were made at the Institute for Low Temperature Physics and Engineering (Kharkov) on a special helium cryostat mounted on a standard DRON-4 x-ray powder diffractometer using Co radiation of the wavelength of $1.79021 \AA$. The cryostat used enabled variation of the temperature of the samples in the interval $4.2-300 \mathrm{~K}$ and stabilization of the temperature values to within $\pm 0.05 \mathrm{~K}$. The samples were obtained by condensation of $\mathrm{SF}_{6}$ gas of purity $99.98 \%$ on a flat copper substrate cooled to $125 \mathrm{~K}$ in the cryostat. The samples were then annealed at $140 \mathrm{~K}$ to eliminate stresses and to reduce the number of defects. It is found that the condensation of $\mathrm{SF}_{6}$ gas onto a lower-temperature substrate produces severely stressed samples, the annealing of which is followed by intense recrystallization and generation of coarse-grained samples, resulting in a lower reliability of the polycrystal x-ray data. The reflections of the analytically pure copper covering the specimen chamber substrate were a modified intrinsic reference for precession estimation of the lattice parameters. The lattice parameters were estimated by the mean-square method at separate temperatures (4.5, 60 , and $78 \mathrm{~K}$ ) with an error of no more than $0.02 \%$. To estimate the parameters, the whole set of experimental x-ray reflections (about 40 reflections) observed in the angular range $2 \Theta=20-91^{\circ}$ was used. The temperature dependence of the lattice parameters in the interval between the above reference points was investigated by measuring the angular positions of the ten most intense reflections. The regions of a possible low-temperature phase transition $(40 \mathrm{~K}<T<60 \mathrm{~K})$ and the well-known hightemperature one (at $94.3 \mathrm{~K}$ ) were studied with a temperature step of $1-2 \mathrm{~K}$, and the intermediate region with a step of $5 \mathrm{~K}$.

\section{Results}

Structure. Our neutron and x-ray diffraction data on the low-temperature phase demonstrate that in the whole temperature range of 1.64 to $94 \mathrm{~K}$ the crystal structure of $\mathrm{SF}_{6}$ undergoes no essential modifications which might be attributed to the second phase transition that, as speculated in [4,5], may occur at $\sim 50 \mathrm{~K}$. To obtain more-convincin evidence, we analyzed the temperature dependences of the half-width and intensity of the most pronounced $x$-ray reflections. If a phase transition did occur, one might expect visible anomalies in the temperature dependences of the $\mathrm{x}$-ray reflection widths or intensities, but the experiments revealed no anomalies. The additional high-symmetry phases observed in [4,5] are most likely nonequilibrium and resulted from either the specific features of the technique of sample preparation or from size effects in the thin films. The authors of [9] also consider that massive equilibrium samples have no intermediate phase of hexagonal symmetry, and the two stages of orientational ordering of molecules observed in [4] owe their origin to the effect of the surface on the kinetics of phase transitions in the thin films of $\mathrm{SF}_{6}$. Thus, our experiments provide support for the neutron-diffraction [13] and the calorimetric [15] results on the unique solid-solid phase transition which occurs at $T=94.3 \mathrm{~K}$.

Of all the experimental data on the structure of the low-temperature phase, we consider the results given in [13] to be the most comprehensive and reliable ones. The diffraction pattern observed in 


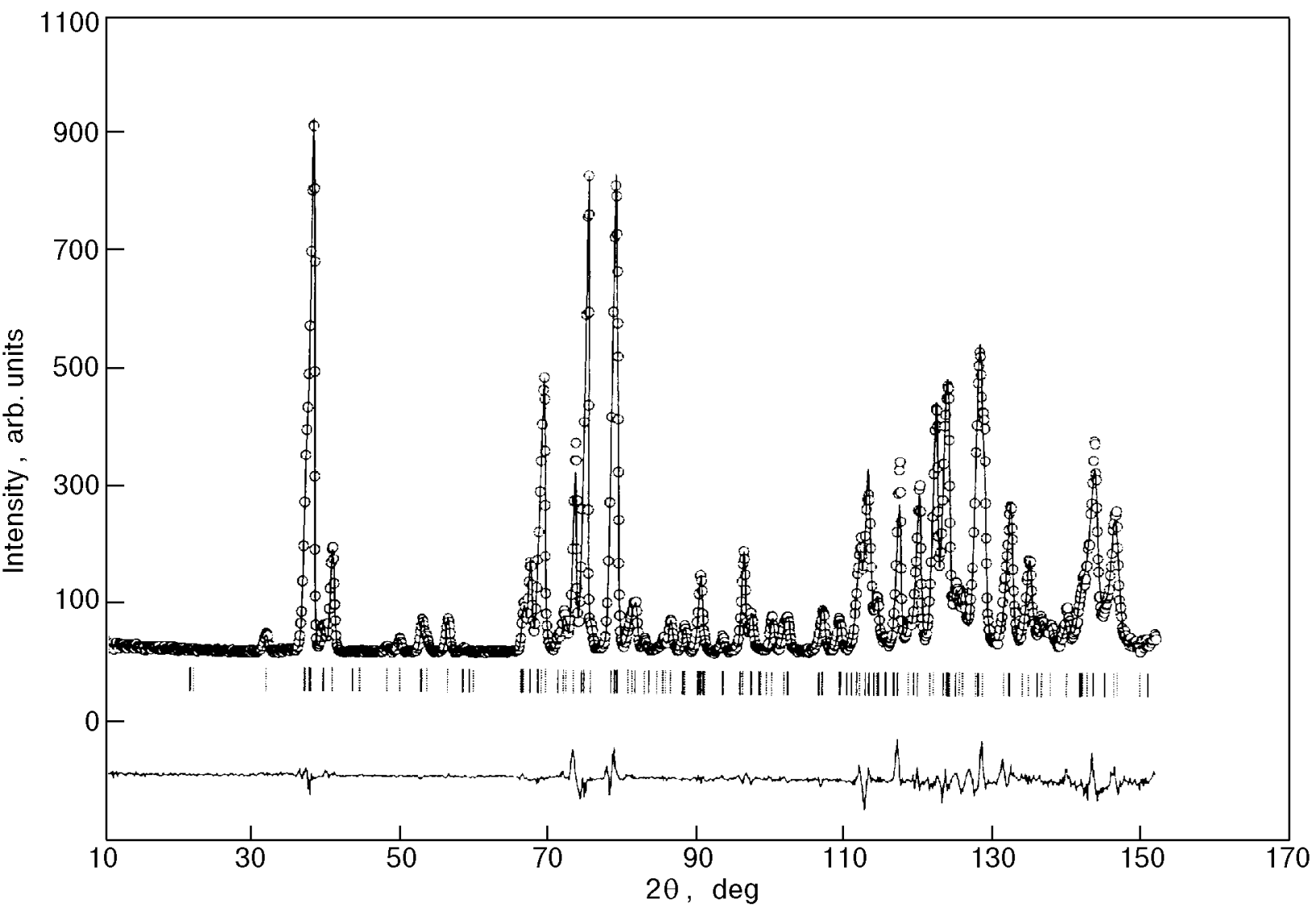

Fig. 1. Observed, calculated, and difference profiles for $\alpha-\mathrm{SF}_{6}$ neutron powder diffraction pattern at $1.64 \mathrm{~K}(\lambda=2.59 \AA)$. Vertical bars indicate the calculated reflection positions.

our neutron-diffraction experiments is qualitatively parison, Figs. 1 and 2 show a neutron $(T=1.64 \mathrm{~K})$ similar to that shown in [13]. For purposes of com- diffraction pattern and $\mathrm{x}$-ray diffraction patterns for

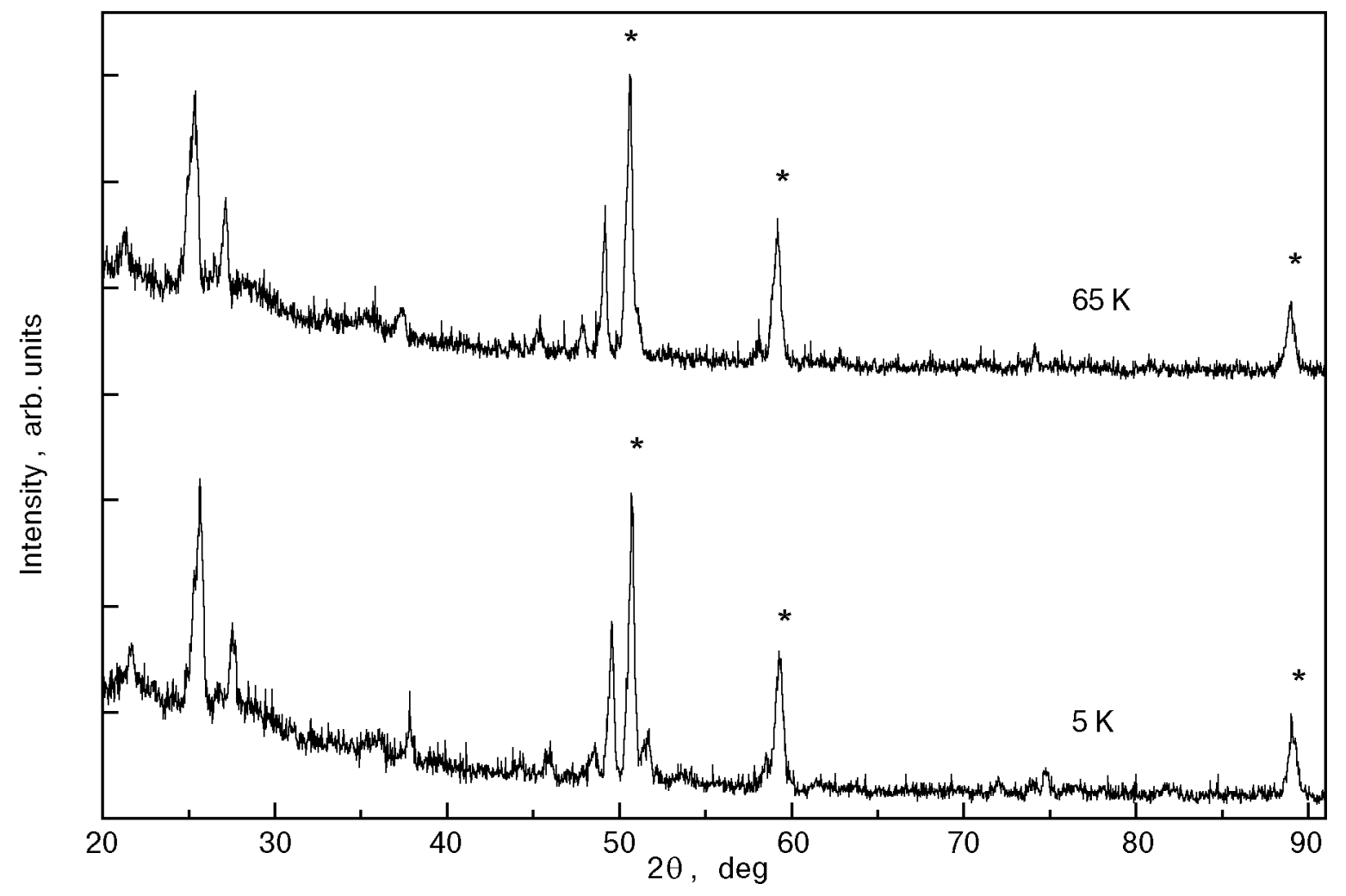

Fig. 2. X-ray powder diffraction pattern of $\alpha-\mathrm{SF}_{6}$ at 5 and $65 \mathrm{~K}(\lambda=1.79021 \AA$ ). Asterisks mark the reference (pure Cu) reflections. 
two temperatures ( 5 and $65 \mathrm{~K}$ ). Since the natures of neutron and $\mathrm{x}$-ray diffraction scattering by crystals are dissimilar, the latter method gives a poorer diffraction pattern. We managed to take only the most pronounced reflections, and a number of doublets and multiplets remained unseparated. Therefore, to index correctly the $\mathrm{x}$-ray reflections, we invoked a visual similarity of the diffraction patterns shown in Figs. 1 and 2. The set of reflections taken is adequately indexed with the assumption of the monoclinic cell proposed in [13]. Using the neutron-diffraction data for $1.64 \mathrm{~K}$, we obtained the following lattice parameters: $a=13.803 \AA$, $b=8.139 \AA, c=4.749 \AA, \beta^{\prime}=95.586^{\circ}$, which are in good agreement within the limits of experimental error with the data of [13] for $T=5 \mathrm{~K}$ and our x-ray data for $T=4.5 \mathrm{~K}$. Recall that the x-ray lattice parameters were found by averaging over the whole set of reflections in a diffraction angle range up to $2 \Theta=91^{\circ}$ by the method of least squares and by refining the true reflection angles as compared to the reference. The $\mathrm{x}$-ray lattice parameters at $4.5 \mathrm{~K}$ were as follows: $a=13.813 \AA, \quad b=8.144 \AA$, $c=4.752 \AA, \beta^{\prime}=95.59^{\circ}$.

The Rietveld analysis of the collected neutron data performed with the use of the FULLPROF program [14] substantiated the suppositions made in [13], according to which the low-temperature monoclinic phase structure of $\mathrm{SF}_{6}$ belongs to the symmetry space group $C 2 m\left(C_{2 h}^{3}\right)$ with $Z=6$. At the first stage of the structural model refinement, isotropic thermal factors with the coefficients $B$ equal to 0.94 and $2.21 \AA^{2}$ for the $\mathrm{S}$ and $\mathrm{F}$ atoms, respectively, were applied. These values were obtained by using the data on the Debye temperature and on the mean-square displacements of molecules in the high-temperature phase [2]. The ultimate Rietveld refinement of the structure at $1.64 \mathrm{~K}$ was also made with the inclusion of an absorption correction and anisotropic thermal factors.

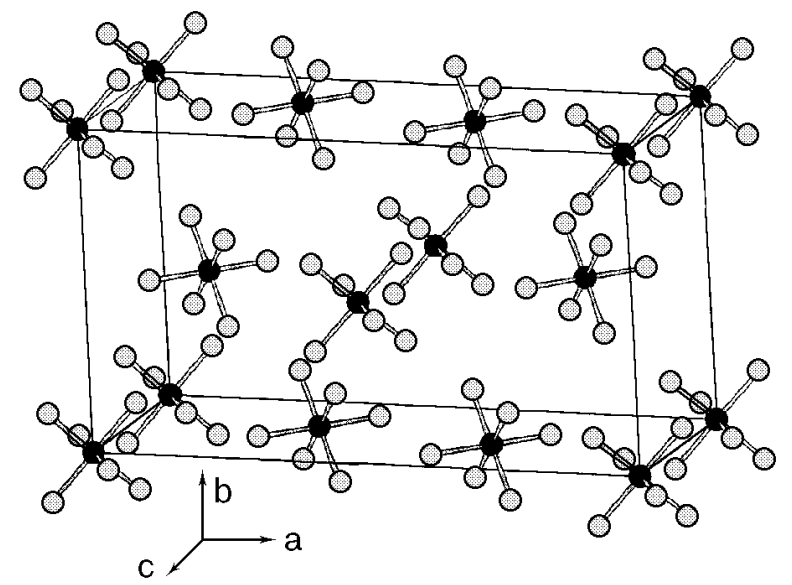

Fig. 3. Monoclinic structure (space group $C 2 / m, Z=6$ ) of the low-temperature phase of $\mathrm{SF}_{6}$.
Unlike the authors of [13], we succeeded already with an isotropic approach and obtained a good agreement between the calculated and measured neutron scattering spectra (see Fig. 1) with a reliability structure factor of $R=3.94 \%$ at $1.64 \mathrm{~K}$ and, besides, with the intramolecular bonds of the $\mathrm{SF}_{6}$ octahedrons remaining undistorted. The molecules held the shape of a regular octahedron with an $\mathrm{S}-\mathrm{F}$ bond length of $1.56 \AA$. Besides, the peakwidth parameters $U, V, W(0.1872,-0.3105$, $0.4807(\mathrm{deg})^{2}$, respectively) resulted in no broadening of the calculated reflection profiles compared to the experimental ones. Table 1 lists the improved parameters of the $\mathrm{SF}_{6}$ structural model for refined isotropic thermal factors at temperatures 1.64 and $75 \mathrm{~K}$. A $3 D$ image of the structure obtained is shown schematically in Fig. 3.

Table 1

The refined structural parameters from neutron scattering data for a powder sample of $\mathrm{SF}_{6}$ at 1.64 and $75 \mathrm{~K}$. Space group $C 2 / m\left(C_{2 h}^{3}\right), Z=6 . x, y, z$ - relative coordinates for atoms

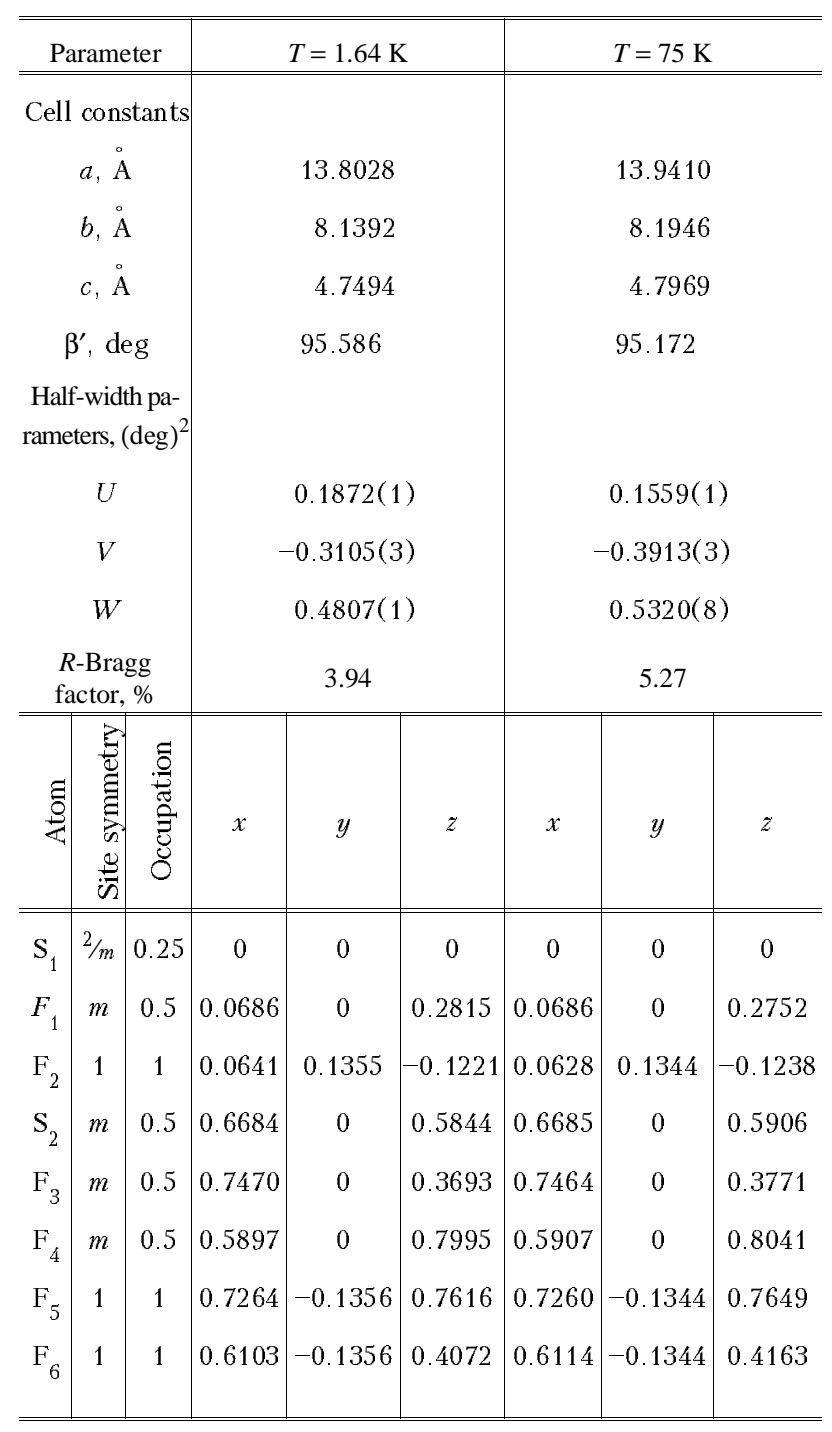




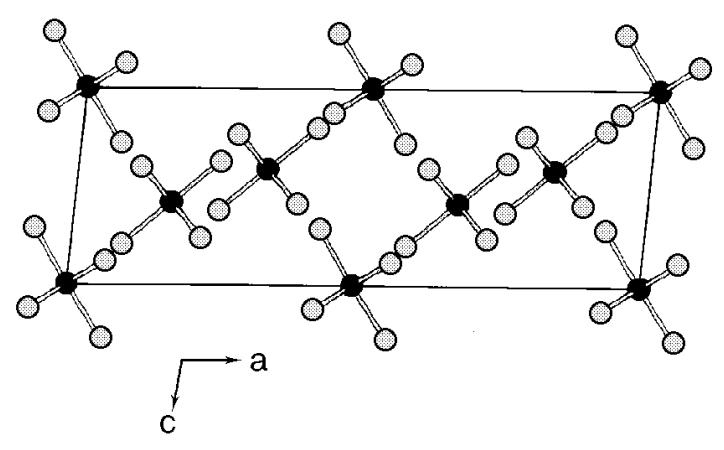

Fig. 4. Unit cell projection of $\alpha-\mathrm{SF}_{6}$ on the monoclinic plane $(a, c)$.

Attention should be drawn to the fact that according to the structure data (Table 1) the $\mathrm{SF}_{6}$ molecules occupy two types of positions in the monoclinic lattice, with dramatically different point symmetry. As the projection of the structure on the monoclinic plane $(a, c)$ shows (Fig. 4), one-third of the molecules, with the $\mathrm{S}$ atoms in the $(0,0,0)$ position, have the highest local symmetry $2 / m$ possible in the space group under consideration. Hence, with increase in temperature the intensity and amplitudes of rotational motion of these molecules is likely to be higher than that for the remaining $2 / 3$ of the molecules, in the position $(x, 0, z)$ with a lower local symmetry $m$. It will be shown below that the presence of these two types of molecules in the structure has the determining influence on the thermodynamic properties of the low-temperature phase of sulphur hexafluoride.

The structural model and the thermal factors $B$ were refined in the isotropic approximation using neutron diffraction data for each temperature studied. As a result, information was derived which describes the temperature variation of the effective isotropic thermal factor $B$ of molecules occupying two different positions. These results are shown in Fig. 5. At low temperatures $(T<40 \mathrm{~K})$ the values of the coefficients $B$ are very low and practically similar for molecules having different local symmetries. Above $40 \mathrm{~K}$ the one-third of the molecules which are at the sites of the higher point symmetry $2 / m$ have larger $B$ values, and we observe a more intensive growth of these values with temperature as compared to the molecules at the sites of symmetry $m$. This behavior of $B(T)$ indicates that rising temperature leads to considerable enhancement of the rotational motion of the molecules at the $2 / \mathrm{m}$ positions. It is possible that the rotational motion becomes nearly free in the vicinity of $T_{c}$. This assumption is supported by our analysis of the thermodynamic characteristics of the low-tempera-

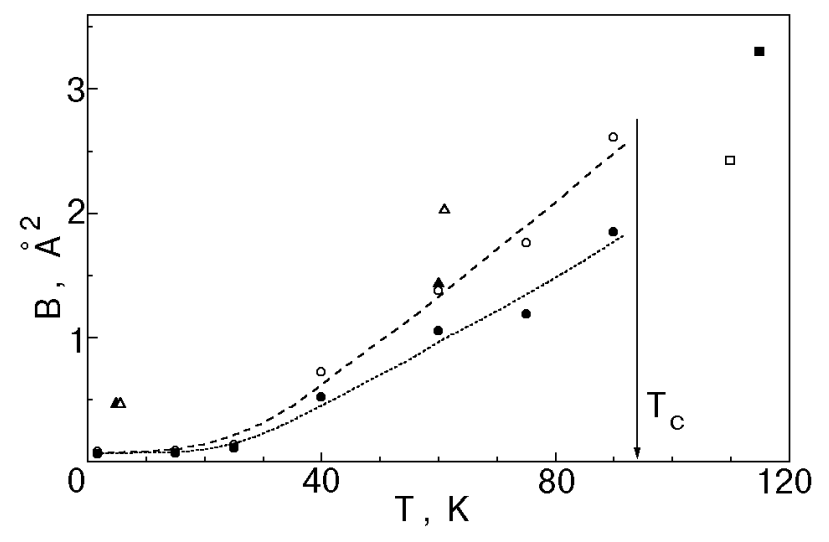

Fig. 5. Temperature change of the isotropic thermal coefficient $B: \bigcirc,-$ - the data for molecules at local symmetry positions $2 / m$ and $m$ in the low-temperature phase, respectively; $\Delta, \boldsymbol{\Delta}$ - the same data calculated with the use of the experimental data of [13]; $\square$ and $\boldsymbol{\square}$ - our previous data from [2] and [13] for the high-temperature phase. $T_{c}$ is the temperature of the solid-solid phase transition.

ture phase. The presence of two types of such molecules in the $\alpha-\mathrm{SF}_{6}$ crystals may be one of the reasons for the appearance of the intermediate metastable phase in specially prepared samples. For example, this may cause the formation of a rhombohedral symmetry phase in thin $\mathrm{SF}_{6}$ films in the range $50 \mathrm{~K}-T_{c}[4,5]$. Using the data of [13], we have calculated the effective values of the isotropic $B$ factor at 5 and $60 \mathrm{~K}$. Along with the data directly measured at $115 \mathrm{~K}$ [13], our calculated results are systematically higher both in the low- and in the high-temperature phases (see Fig. 5). Also of interest is the fact that when using the data of [13], anomalously high $B$ factors appear even at helium temperatures. These values correspond to our present results at $40 \mathrm{~K}$. The probable reason for the observed systematic discrepancy of $B(T)$ may be the difference in the purity and structural perfection of the samples used in these two studies.

Lattice parameters. For the purpose of obtaining data on the thermal expansion of $\alpha-\mathrm{SF}_{6}$, we measured in detail the variations with temperature of the angular positions of the most intense reflections in the $x$-ray diffraction experiments. That made it possible to determine reliably the temperature dependences of the lattice parameters and to estimate the thermal expansion coefficients from them. We found that our x-ray and neutron diffraction data on the lattice parameters agree satisfactorily with each other over the whole temperature existence region of the low-temperature phase and that they also allow us to estimate their consistency with the results given in [13]. The temperature variations of the lattice parameters and the monoclinic angle are shown in Fig. 6, and those of the molar volume and 

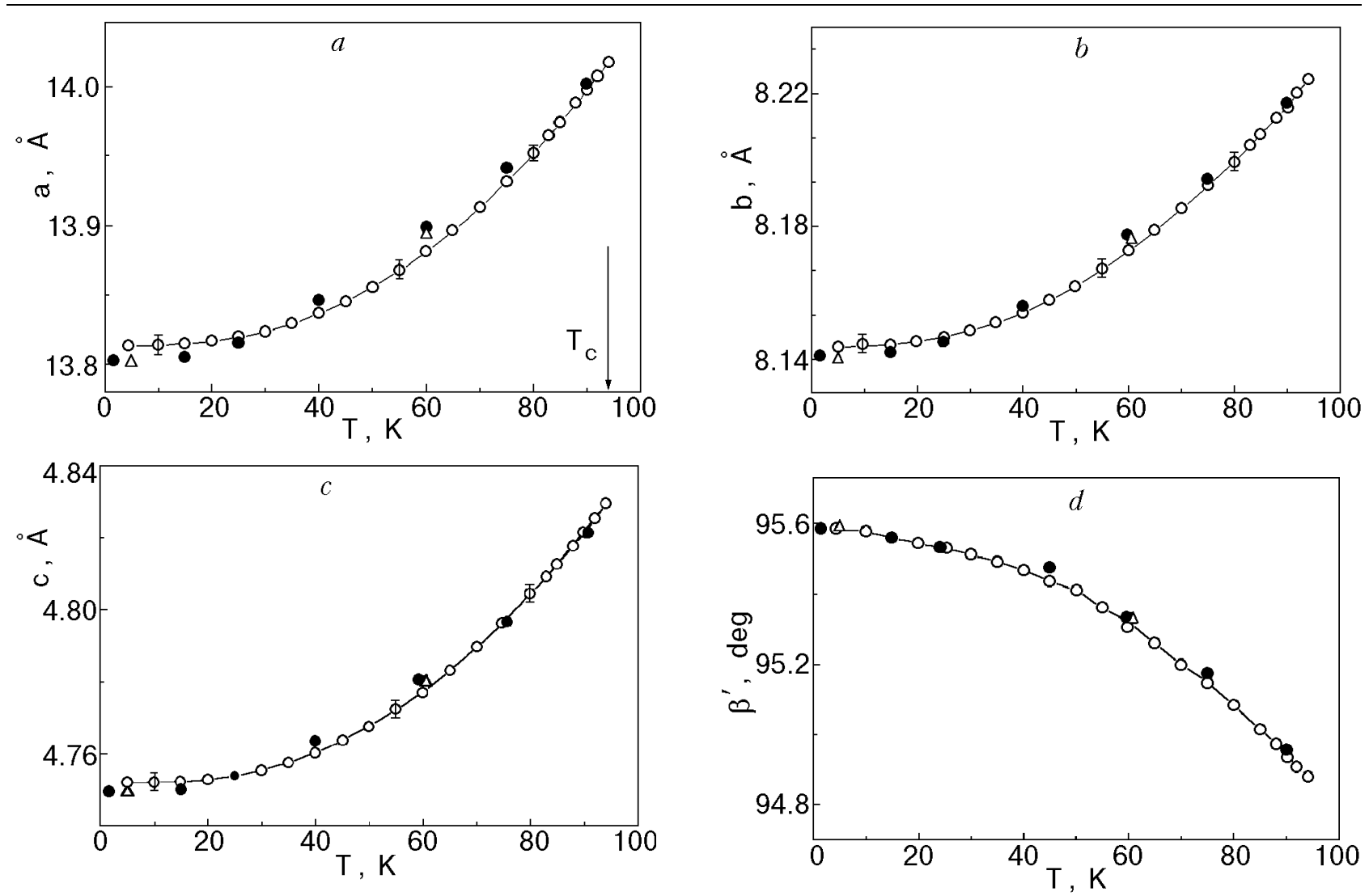

Fig. 6. Temperature dependences of $a(a), b(b), c(c), \beta^{\prime}(d)$, the lattice parameters of monoclinic phase of $\mathrm{SF}_{6}: \bigcirc, \bullet-$ the data obtained in present work by $\mathrm{x}$-ray and neutron methods, respectively; $\Delta$ corresponds to the results obtained in [13]. $T_{c}$ is the temperature of solid-solid phase transition.

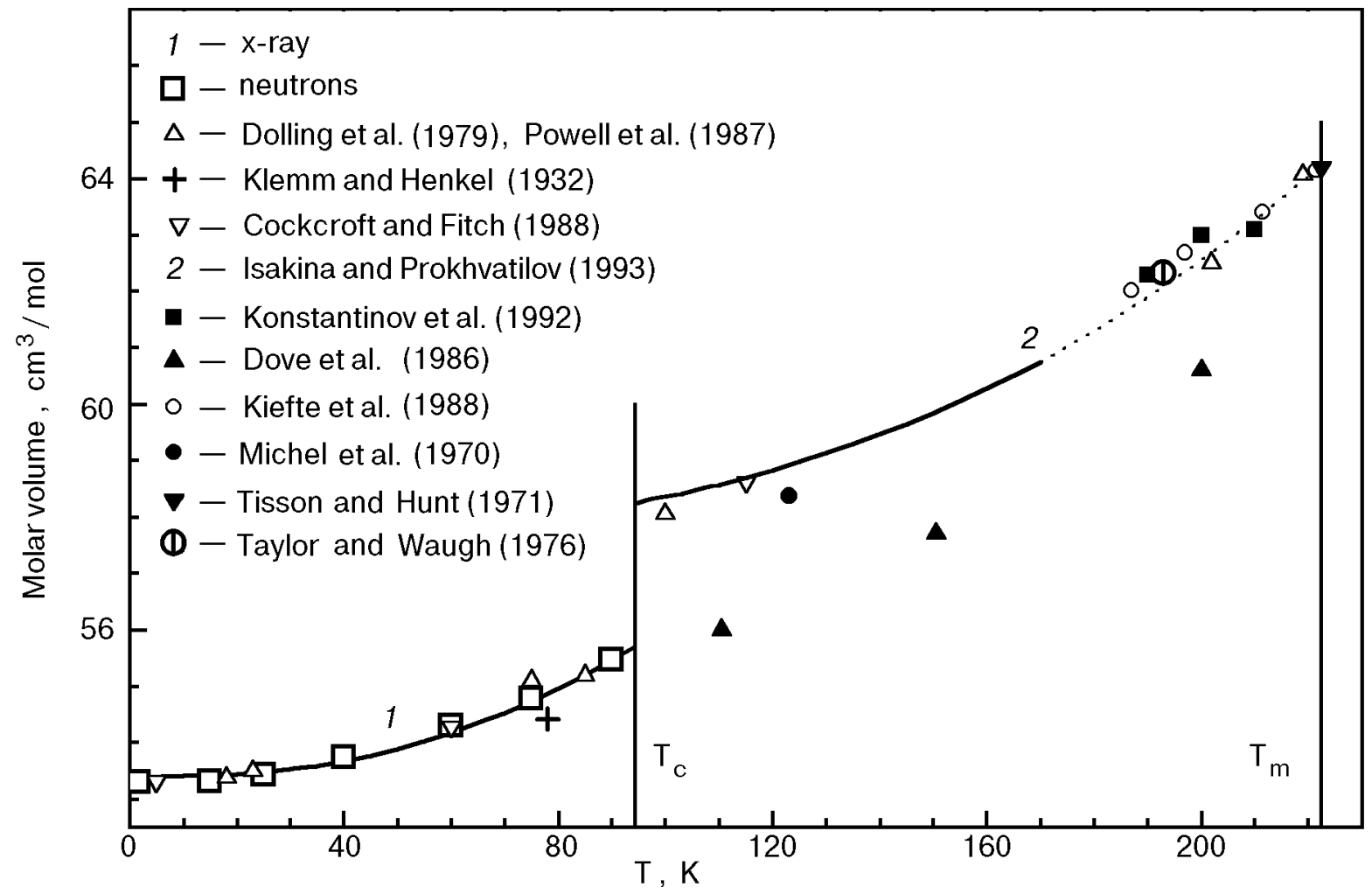

Fig. 7. Temperature dependences of the molar volume of $\mathrm{SF}_{6}$ in the low- and high-temperature phases: 1 and $\square$ show the data obtained in present work; 2 was obtained in Ref. 2, $\bullet$ in Ref. 1, $\Delta$ in Refs. 3, 16, $\nabla$ in Ref. 13, + in Ref. 17, $\boldsymbol{\square}$ in Ref. 18, $\boldsymbol{\Delta}$ in Ref. 19, $O$ in Ref. 20, $\nabla$ in Ref. 21, $\phi$ in Ref. 22. $T_{c}$ and $T_{m}$ correspond to the temperatures of phase transition and melting point, respectively. 
its jump at the phase transition are illustrated in Fig. 7 together with the available data from the literature. The observed slight disagreement of the lattice parameters with the data given in [13] may be attributed to two reasons. The authors of [13] employed a $\mathrm{SF}_{6}$ gas of insufficiently high purity $99.9 \%$ (we used $99.99 \%$ pure gas), resulting, as a rule, in considerable (and in some cases gigantic) anomalies of the physical properties at low temperatures and producing an appreciable effect on the dynamics of the crystal lattices [23]. On the other hand, contrary to the present x-ray and neutron diffraction measurements, the experiments in [13] were performed on a sample that was not free (the sample was grown in a glass ampoule at high temperatures, cooled to $77 \mathrm{~K}$, and then quenched quickly down to liquid-helium temperature), and hence, the sample might have high stresses of the first kind.

Extrapolation of the temperature dependence of the lattice volume to the phase transition temperature $(94.3 \mathrm{~K})$ results in the value $V_{m}=$ $=55.68 \mathrm{~cm}^{3} / \mathrm{mol}$, which nearly agrees with the value $V_{m}=56.0 \mathrm{~cm}^{3} / \mathrm{mol}$ obtained by substracting the volume change at the transition $\left(\Delta V_{m}=\right.$ $=2.1 \mathrm{~cm}^{3} / \mathrm{mol}$ by the estimates in [24]) from the high-temperature phase volume [2]. The value of the jump in volume measured within a narrow temperature range of 94 to $94.5 \mathrm{~K}$ in the vicinity of the phase transition turns out to be somewhat higher than that expected from the estimation by the Clayperon-Clausius equation in [24] and amounts to $2.57 \mathrm{~cm}^{3} / \mathrm{mol}$ (approximately $4.4 \%$ ), but this value is lower than the change in density at the transition (6\%) obtained in the pycnometric measurements reported in [17]. As is evident from Fig. 7, our temperature dependence of the molar volume is in good agreement with all of the experimental and theoretical estimates except for the data of [19], which appear to be much lower. The last fact seems to be responsible for the increased value of the change in density at the transition in [19].

Thermodynamic properties. The temperature dependences of the linear expansion coefficients, illustrating the character of expansion anisotropy in $\alpha-\mathrm{SF}_{6}$, are shown in Fig. 8. Our attention is engaged by the fact that, beginning just with $50 \mathrm{~K}$, the temperature dependences of the linear $\alpha_{a}, \alpha_{c}$ and volume $\beta$ expansion coefficients become more pronounced. As will be show below, this behavior is mainly dictated by the variations in the dynamics of molecular rotational motion in the monoclinic lattice at this temperature. The numerical values of the lattice parameters and molar volume, together with

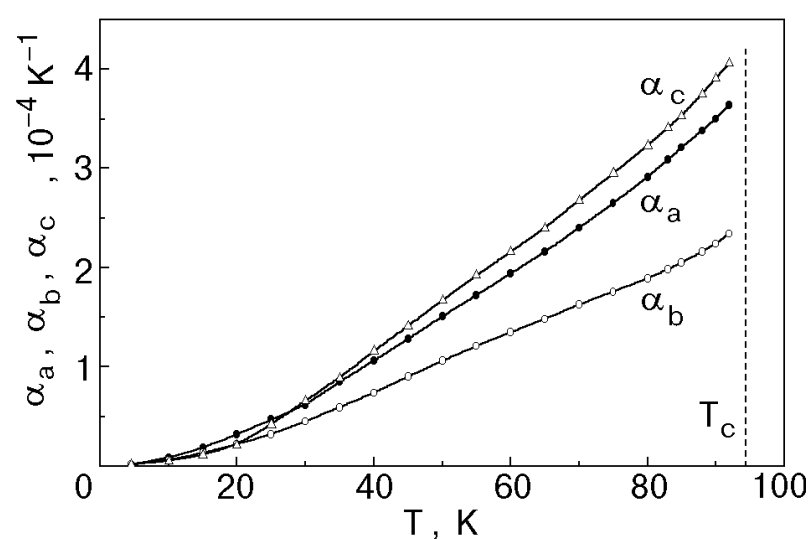

Fig. 8. Linear thermal expansion coefficients versus temperature. $T_{c}$ is the temperature of the solid-solid phase transition.

the thermal expansion coefficients, are listed in Table 2 .

The solid-solid phase transition and the peculiarities of physical properties of $\mathrm{SF}_{6}$ crystals, as of other molecular cryocrystals, are a consequence of variations in the rotational subsystem. Observation and identification of these variations can be done with the method of separating the contributions of phonon and rotational subsystems to the lattice dynamics [26]. The data on the thermal expansion coefficients enabled us to apply this method, along with published data on the heat capacity at constant pressure $C_{P}$ [15], to recognize and to analyze the rotational component of the heat capacity at constant volume $C_{\text {rot }}$.

With allowance made for all type of vibrations in the lattice and their mutual independence being assumed, the heat capacity of a molecular crystal can be given as a sum of contributions from the translational and rotational subsystems and a contribution due to the intramolecular vibrations of the atoms:

$$
C_{V}=C_{\mathrm{tr}}+C_{\text {rot }}+C_{\text {in }} .
$$

The heat capacity at constant volume, $C_{V}$, is difficult to measure. In practice, it is calculated, when data on the thermal expansion and compressibility are available, from the values of the heat capacity at constant pressure, $C_{P}$, by using the known thermodynamic relation:

$$
C_{P}-C_{V}=\beta^{2} V T / \chi_{T} .
$$

The calculations of $C_{V}$ for the low-temperature phase of $\mathrm{SF}_{6}$ were made difficult by the lack of data on the compressibility $\chi_{T}$. The compressibility of $\mathrm{SF}_{6}$ was studied in [24] by the piston displacement method in the 81-190 K temperature and 0-20 kbar 
Table 2

Experimental x-ray $(a)$ and neutron $(b)$ values of the lattice parameters $\left(a, b, c, \beta^{\prime}\right)$, molar volume $\left(V_{m}\right)$, density $(\rho)$, linear $\left(\alpha_{a}\right.$, $\left.\alpha_{b}, \alpha_{c}\right)$, and volume $(\beta)$ thermal expansion coefficients

\begin{tabular}{|c|c|c|c|c|c|c|c|c|c|c|}
\hline \multirow{2}{*}{$T, \mathrm{~K}$} & \multirow{2}{*}{$a, \stackrel{\mathrm{A}}{ }$} & \multirow{2}{*}{$b, \mathrm{~A}$} & \multirow{2}{*}{$c, \stackrel{\AA}{A}$} & \multirow{2}{*}{$\beta^{\prime}, \operatorname{deg}$} & \multirow{2}{*}{$\begin{array}{c}V_{m}, \\
\mathrm{~cm}^{3} / \mathrm{mol}\end{array}$} & \multirow{2}{*}{$\begin{array}{c}\rho, \\
g / \mathrm{cm}^{3}\end{array}$} & $\alpha_{a}$ & $\alpha_{b}$ & $\alpha_{c}$ & $\beta$ \\
\hline & & & & & & & \multicolumn{4}{|c|}{$10^{-4}, \mathrm{~K}^{-1}$} \\
\hline \multicolumn{11}{|l|}{ a) } \\
\hline 4.5 & 13.813 & 8.144 & 4.752 & 95.59 & 53.40 & 2.735 & - & - & - & - \\
\hline 10 & 13.814 & 8.144 & 4.752 & 95.58 & 53.41 & 2.735 & 0.08 & 0.06 & 0.05 & 0.19 \\
\hline 15 & 13.815 & 8.145 & 4.752 & 95.56 & 53.42 & 2.734 & 0.18 & 0.13 & 0.11 & 0.42 \\
\hline 20 & 13.818 & 8.146 & 4.753 & 95.54 & 53.44 & 2.733 & 0.32 & 0.22 & 0.21 & 0.75 \\
\hline 25 & 13.820 & 8.147 & 4.754 & 95.53 & 53.47 & 2.732 & 0.47 & 0.32 & 0.42 & 1.21 \\
\hline 30 & 13.824 & 8.149 & 4.755 & 95.51 & 53.52 & 2.729 & 0.54 & 0.45 & 0.66 & 1.65 \\
\hline 35 & 13.830 & 8.151 & 4.758 & 95.49 & 53.58 & 2.726 & 0.85 & 0.59 & 0.89 & 2.33 \\
\hline 40 & 13.837 & 8.154 & 4.760 & 95.46 & 53.66 & 2.722 & 1.06 & 0.74 & 1.16 & 3.00 \\
\hline 45 & 13.846 & 8.158 & 4.764 & 95.43 & 53.76 & 2.717 & 1.28 & 0.90 & 1.43 & 3.74 \\
\hline 50 & 13.856 & 8.162 & 4.768 & 95.40 & 53.88 & 2.711 & 1.51 & 1.06 & 1.68 & 4.43 \\
\hline 55 & 13.868 & 8.167 & 4.772 & 95.36 & 54.01 & 2.704 & 1.72 & 1.21 & 1.92 & 5.05 \\
\hline 60 & 13.882 & 8.173 & 4.777 & 95.32 & 54.16 & 2.697 & 1.94 & 1.35 & 2.16 & 5.66 \\
\hline 65 & 13.897 & 8.179 & 4.783 & 95.26 & 54.34 & 2.688 & 2.16 & 1.48 & 2.40 & 6.25 \\
\hline 70 & 13.913 & 8.185 & 4.790 & 95.20 & 54.52 & 2.679 & 2.40 & 1.63 & 2.68 & 6.87 \\
\hline 75 & 13.932 & 8.192 & 4.797 & 95.15 & 54.73 & 2.669 & 2.65 & 1.76 & 2.95 & 7.54 \\
\hline 80 & 13.952 & 8.200 & 4.804 & 95.08 & 54.95 & 2.658 & 2.91 & 1.89 & 3.23 & 8.18 \\
\hline 83 & 13.965 & 8.205 & 4.809 & 95.04 & 55.09 & 2.651 & 3.09 & 1.98 & 3.41 & 8.62 \\
\hline 85 & 13.974 & 8.208 & 4.813 & 95.01 & 55.19 & 2.649 & 3.21 & 2.05 & 3.53 & 8.91 \\
\hline 88 & 13.988 & 8.213 & 4.818 & 94.97 & 55.34 & 2.639 & 3.38 & 2.16 & 3.75 & 9.36 \\
\hline 90 & 13.998 & 8.217 & 4.821 & 94.94 & 55.45 & 2.634 & 3.50 & 2.24 & 3.91 & 9.68 \\
\hline 92 & 14.008 & 8.220 & 4.825 & 94.91 & 55.56 & 2.629 & 3.64 & 2.34 & 4.06 & 10.02 \\
\hline 94 & 14.017 & 8.224 & 4.829 & 94.88 & 55.67 & 2.623 & - & - & - & - \\
\hline \multicolumn{11}{|l|}{ b) } \\
\hline 1.64 & 13.8028 & 8.1392 & 4.7494 & 95.586 & 53.301 & 2.7401 & & & & \\
\hline 15 & 13.8053 & 8.1399 & 4.7500 & 95.560 & 53.323 & 2.7390 & & & & \\
\hline 25 & 13.8165 & 8.1446 & 4.7539 & 95.530 & 53.438 & 2.7331 & & & & \\
\hline 40 & 13.8463 & 8.1561 & 4.7633 & 95.475 & 53.748 & 2.7173 & & & & \\
\hline 60 & 13.8976 & 8.1772 & 4.7805 & 95.330 & 54.294 & 2.6900 & & & & \\
\hline 75 & 13.9410 & 8.1946 & 4.7969 & 95.172 & 54.780 & 2.6662 & & & & \\
\hline 90 & 14.0024 & 8.2174 & 4.8210 & 94.952 & 55.468 & 2.6330 & & & & \\
\hline
\end{tabular}

pressure ranges. But in the above paper primary attention was given to the $P-T$ diagram and the phase transition characteristics. A numerical value of the isothermal compressibility was cited only for the high-temperature phase at $T=182 \mathrm{~K}$ $\left(\chi_{T}=2.93 \cdot 10^{5} \mathrm{~cm}^{2} / \mathrm{kg}\right)$. In a previous paper [2] in order to estimate compressibility everywhere over the existence region of the high-temperature phase $(95-220 \mathrm{~K})$ we used the data on the elastic constant obtained from an investigation of the Brillouin light scattering in $\mathrm{SF}_{6}$ single crystals [20]. In the present paper these results, with regard to their variations at the phase transition due to the large change in volume were extrapolated to the existence region of the low-temperature phase. Moreover, it was assumed that at low temperatures the linear, though rather weak, dependence of the compressibility still persists. The temperature dependences of the heat capacity $C_{V}$ thus calculated are shown in Fig. 9. The numerical values of the other thermodynamic characteristics of the low-temperature phase of $\mathrm{SF}_{6}$ are also listed in Table 3.

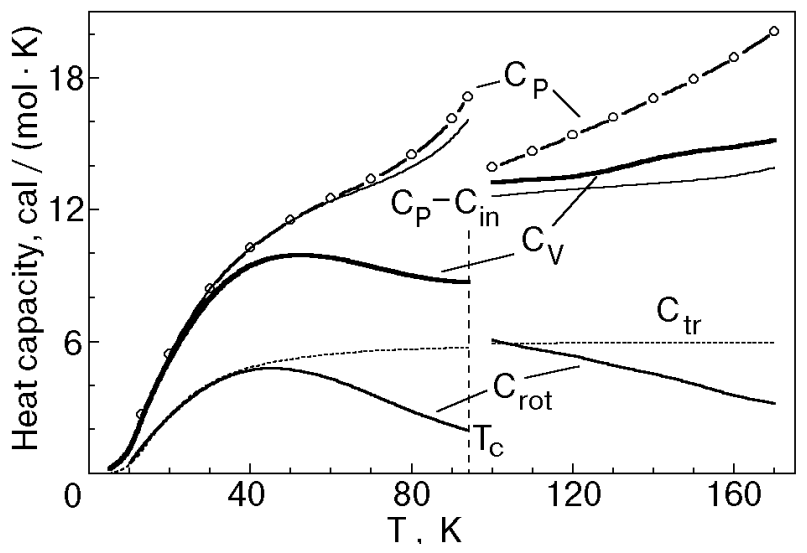

Fig. 9. Temperature dependence of the heat capacity contributions in $\alpha-\mathrm{SF}_{6}: O$ show the data [14] on the heat capacity at constant pressure, $C_{P}$, while $C_{V}, C_{\text {in }}, C_{\text {tr }}$, and $C_{\text {rot }}$ correspond to the calculated curves for the heat capacity at constant volume and for the intramolecular, translational, and rotational components of the heat capacity, respectively. 
The heat capacity $C_{\text {in }}$ was calculated from the characteristic temperatures $\Theta_{i}=h v_{i} / k$ in the Einstein model approximation with the use of the intramolecular vibrational frequencies $v_{i}$ cited in [27]. The contribution of translational vibrations to the heat capacity was calculated in the Debye approximation with a characteristic temperature $\Theta_{D}=65 \mathrm{~K}$ obtained from an analysis of the lowtemperature part of the heat capacity [15]. As is evident from Fig. 9, the temperature dependence of $C_{\mathrm{tr}}$ decreases distinctly even at $T>30 \mathrm{~K}$, and in the vicinity of the phase transition temperature the heat capacity values almost approach the high-temperature limit $3 R=5.97 \mathrm{cal} /(\mathrm{mol} \cdot \mathrm{K})$.

The heat capacity of a librational subsystem $C_{\text {rot }}=C_{V}-C_{\mathrm{tr}}-C_{\mathrm{in}}$ exhibits an extraordinary temperature dependence (Fig. 9). Considering that the low-temperature phase of $\mathrm{SF}_{6}$ is completely orientationally ordered, for a system of three-dimensional rotators (librators) one may expect that the heat capacity $C_{\text {rot }}$ increases monotonically with temperature and, within the limit of free spherical rotation of molecules, may be close to $3 R$ as the phonon heat capacity. Instead, one observes a reduction in the rotational component at $T>50 \mathrm{~K}$. Such behavior is usually observed only at rather high (pre-melting) temperatures for cryocrystals and may be a result of our not taking into account the anharmonicity of the angular and translational vibrations of the molecules [26]. A noticeable an- harmonicity of the translational vibrations is unlikely at such low temperatures $\left(T<0.5 T_{\mathrm{tr}}\right.$, where $T_{\mathrm{tr}}$ is the triple-point temperature). Therefore, the reduction in the heat capacity is most likely to be due to the unhindering of the molecular rotational motion of one-third of the molecules in the $\alpha-\mathrm{SF}_{6}$ lattice to almost a free rotation, as is also confirmed by the data given in [28]. For the above molecules the heat capacity value may not exceed $(3 / 2) R / 3$. Then for the vibrational subsystem in which twothirds of the molecules execute librational motion and one-third rotate freely, the total heat capacity in the vicinity of the phase transition can't exceed $2.5 R$, in agreement with the results of our calculations (Fig. 9). Thus, the reduction in $C_{\text {rot }}$ observed at $T>50 \mathrm{~K}$ may be accounted for as being due to two sources. First, the heat capacity of the rotational subsystem decreases appreciably because of the unhindering of the molecular rotation from the strongly correlated motion to an almost free one. Secondly, the rotational anharmonicities are strongly enhanced as the phase transition point is approached. A similar behavior of the rotational heat capacity has also been observed in the high-temperature phase of $\mathrm{SF}_{6}$ [2] (see Fig. 9). The numerical values of the thermodynamic characteristics are listed in Table 3.

The Grüneisen constants, which are a measure of the lattice vibrational anharmonicity and its change with increase in temperature, presuppose, in the

Table 3

Adiabatic compressibility $\left(\chi_{S}\right)$, Grüneisen constant $(\gamma)$, heat capacity at constant pressure $\left(C_{P}\right)$ and constant volume $\left(C_{V}\right)$, intramolecular $\left(C_{\text {in }}\right)$, lattice $\left(C_{\text {tr }}\right)$ and librational $\left(C_{\text {rot }}\right)$ components of the heat capacity of sulphur hexafluoride in the low-temperature phase

\begin{tabular}{|c|c|c|c|c|c|c|c|}
\hline \multirow{2}{*}{$T, \mathrm{~K}$} & \multirow{2}{*}{$\begin{array}{c}\chi_{S}, 10^{-11} \\
\mathrm{~cm}^{2} / \mathrm{dyn}\end{array}$} & \multirow{2}{*}{$\gamma$} & $C_{P}[14]$ & $C_{V}$ & $C_{\text {in }}$ & $C_{\mathrm{tr}}$ & $C_{\text {rot }}$ \\
\hline & & & \multicolumn{5}{|c|}{$\mathrm{cal} /(\mathrm{mol} \cdot \mathrm{K})$} \\
\hline 13 & 0.676 & 2.39 & 2.68 & 2.68 & $10^{-7}$ & 1.05 & 1.66 \\
\hline 20 & 0.685 & 2.58 & 5.41 & 5.39 & $10^{-6}$ & 2.69 & 2.75 \\
\hline 30 & 0.698 & 3.59 & 8.41 & 8.26 & $10^{-4}$ & 4.09 & 4.18 \\
\hline 40 & 0.712 & 5.25 & 10.26 & 9.65 & $10^{-3}$ & 4.83 & 4.82 \\
\hline 50 & 0.730 & 6.77 & 11.52 & 10.02 & 0.032 & 5.19 & 4.80 \\
\hline 60 & 0.748 & 7.84 & 12.51 & 9.88 & 0.109 & 5.41 & 4.36 \\
\hline 70 & 0.769 & 8.71 & 13.39 & 9.44 & 0.271 & 5.55 & 3.61 \\
\hline 80 & 0.788 & 9.40 & 14.49 & 8.97 & 0.530 & 5.64 & 2.80 \\
\hline 90 & 0.812 & 9.78 & 16.15 & 8.78 & 0.894 & 5.71 & 2.18 \\
\hline 94 & 0.822 & 9.81 & 17.14 & 8.75 & 1.067 & 5.73 & 1.95 \\
\hline
\end{tabular}


quasi-harmonic approximation, a homogeneous distortion of the spectrum with variation in volume and are given by the relation:

$$
\gamma_{i}=-d \ln \omega_{i} / d \ln V,
$$

where $\omega_{i}$ are the lattice vibration frequencies and $V$ is the crystal volume. From the Grüneisen law there follows a simple thermodynamic relation for calculation of the Grüneisen constants (parameters), which relates the fundamental thermodynamic characteristics of the material:

$$
\gamma=\beta V / \chi_{T} C_{V}
$$

For the most cryocrystals at low temperatures, where the effect of the librational subsystem is insignificant, the values of $\gamma$ are within the range 2.2-2.9 [26]. Also within this range are the values of the Grüneisen parameters for the high-temperature phase of $\mathrm{SF}_{6}$ obtained in [2]. The librational component of $\gamma$ which defines the anharmonicities in the librational subsystem is usually somewhat lower and equals 1.6-1.8 [26]. In our work we did not separate the lattice and librational contributions to the Grüneisen parameters because there are no experimental data on the temperature and volume dependences of the librational vibration spectrum.

The Grüneisen parameters calculated by Eq. (4) are shown in Fig. 10 along with the data for the high-temperature phase. Mention should be made of the unexpectedly considerable change in $\gamma$ at the phase transition and the high values of the constants $\gamma$ for the low-temperature phase as compared to the data for the cryocrystals known to us. The rapid increase in $\gamma$ as the phase transition temperature $T_{c}$ is approached may be responsible for the fact that the anharmonicity of the librations and the orientational disordering of the $\mathrm{SF}_{6}$ molecules have a more distinct influence on the thermal expansion

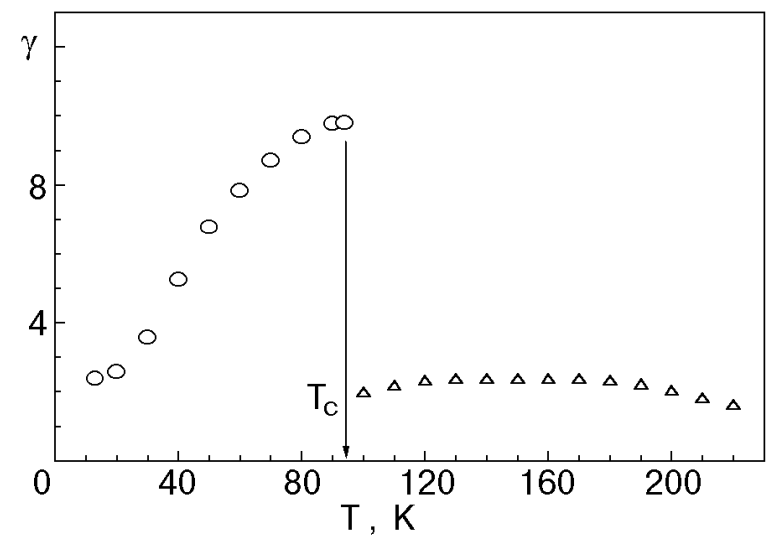

Fig. 10. Grüneisen parameters for the low- and high-temperature phases of $\mathrm{SF}_{6}$ : $\bigcirc$ show the data of the present work, $\Delta$ were obtained in Ref. 2. coefficients of the crystal than on its heat capacity, as follows from Eq. (4) and the experimental dependence of the volume expansion coefficient $\beta$ (see Table 2).

Attention should be paid to the fact that the analysis of the low-temperature heat capacity at $T<20 \mathrm{~K}$ resulted in comparable phonon and librational contributions (within the limits of error). This result is physically unwarranted and may be a consequence of too-high experimental values of $C_{P}$ [15] at the lowest temperatures of their estimation and also due to the lack of measurements of the heat capacity at temperatures below $13 \mathrm{~K}$. The too-high values of the low-temperature heat capacity may have resulted from impurities in the samples. As mentioned above, even insignificant amounts of impurities (less than $0.01 \%$ ) have an appreciable effect on the absolute values and behavior of the heat capacity of simple molecular crystals, particularly at low temperatures [23]. The lack of data on the heat capacity at $T<13 \mathrm{~K}$ makes it difficult to estimate of a low-temperature value of the zero Debye temperature $\Theta_{0}$. We believe, however, that no fundamental error has been made because the calculations were performed with $\Theta_{0}=$ $=65 \mathrm{~K}$, somewhat higher, as it should be, than the reliable value of the high-temperature Debye temperature, equal to $\Theta_{\infty}=62 \mathrm{~K}$ [2]. If the calculations of $C_{\operatorname{tr}}$ are performed with higher values of $\Theta_{0}$, we shall obtain entirely unreasonable results - at $T<20 \mathrm{~K}$ the librational contribution to the heat capacity is much higher than the translational one.

In conclusion, the results of a thermodynamic analysis of heat capacity are consistent with the data on the crystal structure of $\alpha-\mathrm{SF}_{6}$ given in [13] and in the present paper as well as with the theoretical deductions in $[10,11]$. These results also support the assumption [28] that the peculiarities in the temperature dependences of the NMR linewidth and second moment observed in the $45-50 \mathrm{~K}$ temperature range are due not to the two-phase state of the samples used but to the fact that the $\mathrm{SF}_{6}$ molecules occupy two types of positions in the lattice of the low-temperature phase and that these positions differ considerably in symmetry, which determines their rotational mobility. According to the data given in [28] the molecular orientation barriers in the two lattice positions differ by a factor of 1.5 and are equal to 1.94 and $2.8 \mathrm{kcal} / \mathrm{mol}$ for the high- and low-symmetry states, respectively. Gaining more-comprehensive information on the dynamics of rotational molecular motion in $\alpha-\mathrm{SF}_{6}$ will require supplementary investigation of the vibrational spectra by Raman and infrared spectroscopy 
techniques, sound velocity measurements, and careful study of the heat capacity, particularly at the lowest temperatures and on high purity samples.

The authors would like to thank the Office of the Laboratory Leon Brillouin of CEA Saclay, France, for support of this neutron diffraction research under experimental grant No 4142-1996.

1. J. Michel, M. Drifford, and P. Rigny, J. Chem. Phys. 67, 31 (1970).

2. A. P. Isakina and A. I. Prokhvatilov, Low Temp. Phys. 19, 142 (1993) [A. P. Isakina and A. I. Prokhvatilov, Fiz. Nizk. Temp. 19, 201 (1993)].

3. G. Dolling, B. M. Powell, and V. F. Sears, Mol. Phys. 37, 1859 (1979)

4. R. Raynard, G. J. Tatlock, and J. A. Venables, Acta Crystallogr. B38, 1896 (1982).

5. R. Raynard and J. A. Venables, Ultramicroscopy 23, 433 (1987).

6. L. S. Bartell, E. J. Valente, and J. C. Caillat, J. Phys. Chem. 91, 2498 (1987)

7. M. T. Dove and G. S. Pawley, J. Phys. C: Solid State Physics 16, 5969 (1983).

8. M. T. Dove and G. S. Pawley, J. Phys. C: Solid State Physics 17, 6581 (1984).

9. B. M. Powell and G. Dolling, Can. J. Chem. 66, 897 (1988).

10. G. S. Pawley and G. W. Thomas, Phys. Rev. Lett. 48, 410 (1982).

11. G. S. Pawley and M. T. Dove, Chem. Phys. Lett. 99, 45 (1983).

12. L. S. Bartell, J. C. Caillat, and B. M. Powell, Science 236, 1463 (1987).
13. J. K. Cockcroft and A. N. Fitch, Z. Kristallogr. 184, 123 (1988).

14. J. Rodriguez-Carvajal, Proc. Satellite Meeting of the XVth Congr. of Int. Union of Crystallography (1986), p. 127.

15. A. Eucken and F. Schroder, Z. Physik. Chemie A41, 307 (1938).

16. B. M. Powell, M. Dove, G. S. Pawley, and L. S. Bartell, Mol. Phys. 62, 865 (1987).

17. W. K. Klemm and P. Henkel, Z. Anorg. Chem. 207, 73 (1932).

18. V. A. Konstantinov, V. G. Manzhelii, and S. A. Smirnov, Sov. J. Low Temp. Phys. 18, 902 (1992) [V. A. Konstantinov, V. G. Manzhelii, and S. A. Smirnov, Fiz. Nizk. Temp. 18, 1190 (1992)].

19. M. T. Dove, D. Fincham, and R. E. Hubbard, J. Mol. Graphics 4, 79 (1986).

20. H. Kiefte, R. Renney, and M. J. Clouter, J. Chem. Phys. 88, 5846 (1988).

21. J. K. Tisson and F. L. Hunt, J. Chem. Phys. 54, 1256 (1971).

22. J. C. Taylor and A. B. Waugh, J. Solid State Chem. 18, 241 (1976)

23. Physics of Cryocrystals, V. G. Manzhelii and Yu. A. Freiman (eds.), AIP Press, New York (1996).

24. J. W. Stewart, J. Chem. Phys. 36, 400 (1962).

25. B. M. Powell and G. Dolling, Mol. Cryst. Liq. Cryst. 52, 27 (1979).

26. Kriokristally, B. I. Verkin and A. F. Prikhot'ko (eds.), Naukova Dumka, Kiev (1983), in Russian.

27. K. S. Krasnov, V. S. Timoshin, T. E. Danilova, and S. B. Khondozhko, in: Molecular Constants for Inorganic Compounds, Khimiya, Leningrad (1968), in Russian.

28. S. K. Garg, J. Chem. Phys. 66, 2517 (1977). 